\title{
72. Synergistic Inhibition of the Growth of Adenocarcinoma 755 by the Combination of Interleukin-2 and Interferon- $\beta$
}

\author{
By Masaaki IIgo,*) Masanori SAKURAI,**) Mikako SHimizU,***) \\ Toshifumi IIzUKA,***) Nagahiro SAIJo,**) and Akio HoSHI*) \\ (Communicated by Takashi Sugimura, M. J. A., Sept. 12, 1986)
}

Introduction. Interleukin-2 (IL-2) is a secretory product of activated helper T-lymphocytes. It has been demonstrated that IL-2 is essential not only for expansion of antigen-triggered T-lymphocytes and cytotoxic $\mathrm{T}$ cells, but also for the activation of natural killer (NK) cells, which have an inhibitory effect on tumor metastasis. Currently there is a great deal of interest in using IL-2 in clinical cancer therapy because large amounts of recombinant human IL-2 (rHIL-2) are available as a result of advances in the field of genetic engineering. Now it is possible for us to use sufficient quantities of rHIL-2 to conduct properly controlled clinical trials. In animal studies, it has been reported that rHIL-2 together with lymphokine activated killer (LAK) cells induces marked decrease in the numbers of established pulmonary metastasis of MCA-105 sarcoma cells in C57BL/6 mice although rHIL-2 alone shows little effect in this experimental system. ${ }^{1)}$ We report here that the growth of adenocarcinoma 755 was markedly inhibited by a combination of rHIL-2 and recombinant murine interferon $-\beta$ (rIFN- $\beta$ ) in C57BL/6 mice, though $\mathrm{rHIL}-2$ or rIFN- $\beta$ alone showed little effect on the tumor growth.

Materials and methods. Specific pathogen-free (SPF) male C57BL/6 mice were obtained from the Shizuoka Laboratory Animal Center (Hamamatsu). Male $\mathrm{nu} / \mathrm{nu}$ mice (BALB/c background) were supplied by CLEA Japan. The mice housed under SPF conditions were used at the age of $6(\mathrm{C} 57 \mathrm{BL} / 6)$ and $10(\mathrm{nu} / \mathrm{nu})$ weeks at the beginning of the experiments. Adenocarcinoma 755, which is syngeneic with the C57BL/6 mouse, was used for this study. Lyophilized anti-asialo GM1 antiserum (Wako Pure Chemical Industries, Osaka) was reconstituted with balanced salt solution. As reported previously, ${ }^{2)}$ when $500 \mu \mathrm{g}$ of anti-asialo GM1 antibody was administered intravenously into the tail vein of $\mathrm{C} 57 \mathrm{BL} / 6$ mice, the function of $\mathrm{NK}$ cells was suppressed. In this study we injected intravenously $1 \mathrm{mg}$ of anti-asialo GM1 antibody on days 5 and 9. Lyophilized human recombinant interleukin-2 (rHIL-2) (specific activity, $1 \times 10^{7}$ units $/ \mathrm{mg}$ of protein) was obtained from Biogen S.A. Company, Switzerland, and Shionogi \& Co., Osaka. Mouse recombinant interferon- $\beta$ (rIFN- $\beta$ ) (specific activity, $5.5 \times 10^{\top}$ international units $/ \mathrm{mg}$ of protein) was supplied by Toray Industries, Inc., Kamakura.

Groups of $8(\mathrm{C} 57 \mathrm{BL} / 6)$ or 4 (nude) mice were used. Adenocarcinoma 755 $\left(5 \times 10^{5}\right.$ cells/mouse) was implanted subcutaneously (s.c.) into the right thigh

*) Pharmacology Division, National Cancer Center Research Institute, Tsukiji, Chuo-ku, Tokyo 104, Japan.

**) Department of Internal Medicine, National Cancer Center Hospital.

***) Department of Surgery, National Cancer Center Hospital. 
on day 0 . The mice were given rHIL-2 $\left(1 \times 10^{5}\right.$ units/mouse) and $\mathrm{rIFN}-\beta$ $\left(1 \times 10^{5}\right.$ international units/mouse $) 8$ times by s.c. injection into the opposite side (left thigh) in the advanced tumor system (days 5-12). The growth of s.c.-implanted adenocarcinoma 755 was monitored by measurement of the perpendicular diameters with calipers, and tumor volume $\left(\mathrm{mm}^{3}\right)$ was calculated by the formula $1 / 2 \times$ (major diameter in $\mathrm{mm}) \times(\text { minor diameter in } \mathrm{mm})^{2}$.

Results and discussion. rHIL-2 alone was found to have a weak inhibitory effect on the growth of s.c.-implanted adenocarcinoma 755, and rIFN- $\beta$ showed no effect. However, the combination of rHIL-2 and rIFN- $\beta$ showed a marked inhibitory effect on tumor growth (Fig. 1). On the 14th day after im-

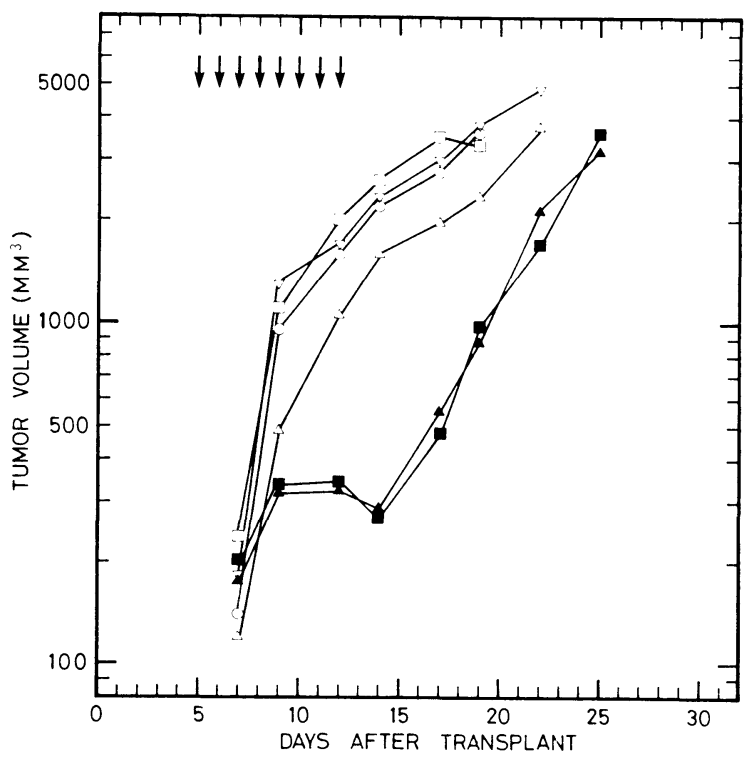

Fig. 1. Inhibitory effect of rHIL-2 and/or rIFN- $\beta$ on tumor growth (adenocarcinoma 755) in C57BL/6 mice. Arrows indicate the treatments of rHIL-2 and/or rIFN- $\beta$. Anti-asialo GM1 antibody was treated on days 5 and 9. Values represent the mean of 8 mice; the S.E.s of the mean were less than $35 \%$ of the mean values. Inhibition in combination groups on 12 through 22 days after transplant was significantly greater than that in rHIL-2 group $(\mathrm{P}<0.05)$. $-\mathrm{O}-$, untreated control $;-\triangle-$, rHIL-2; $-\nabla-$, rIFN- $\beta ;-\triangle-$, rHIL-2+rIFN- $\beta,-\square-$, anti-asialo GM1 antibody; - - - rHIL-2+rIFN- $\beta+$ anti-asialo GM1 antibody.

plantation of tumor, the tumor volumes were $2202 \pm 314$ (S.E.), $1536 \pm 235$ $(\mathrm{T} / \mathrm{C}=68 \%), \quad 2226 \pm 190 \quad(\mathrm{~T} / \mathrm{C}=101 \%), \quad$ and $296 \pm 28 \mathrm{~mm}^{3} \quad(\mathrm{~T} / \mathrm{C}=13 \%)$ $(\mathrm{P}<0.001$, different from rHIL-2 alone) for untreated control, rHIL-2, rIFN- $\beta$, and the combination of rHIL-2 and rIFN- $\beta$, respectively. rHIL-2 and rIFN- $\beta$ are both potent inducers of NK cell activity in mouse cytotoxic T-cell lines, ${ }^{3,4)}$ and synergistic induction of NK activity is obtained by the combination of IL-2 and IFN- $\beta .{ }^{5)}$ Various recent reports suggest that NK cells play an important role in resistance against tumor development in vivo ${ }^{6), 7)}$ Anti-asialo GM1 antibody suppresses the NK activity. ${ }^{2), 8)}$ However, anti-asialo GM1 antibody treatment did not influence the inhibitory effect of the combination of rHIL-2 and 
rIFN- $\beta$ on tumor growth. This suggests that inhibition of tumor growth is primarily mediated by a mechanism that is independent of augmentation of NK cells. Kuribayashi et al.9) showed a spleen cell population exposed to IFN inducer had a markedly augmented ability to bind IL-2, suggesting that IFN causes an increased number and/or affinity of IL-2 receptors. To investigate the participation of T-cell in the potentiation of the inhibition of tumor growth, rHIL-2 and rIFN- $\beta$ were injected s.c. into nude $(\mathrm{nu} / \mathrm{nu})$ mice with adenocarcinoma 755. The results show that the combination of rHIL-2 and rIFN- $\beta$ had an additive, not synergistic, effect (Fig. 2) unlike that in C57BL/6 mice.

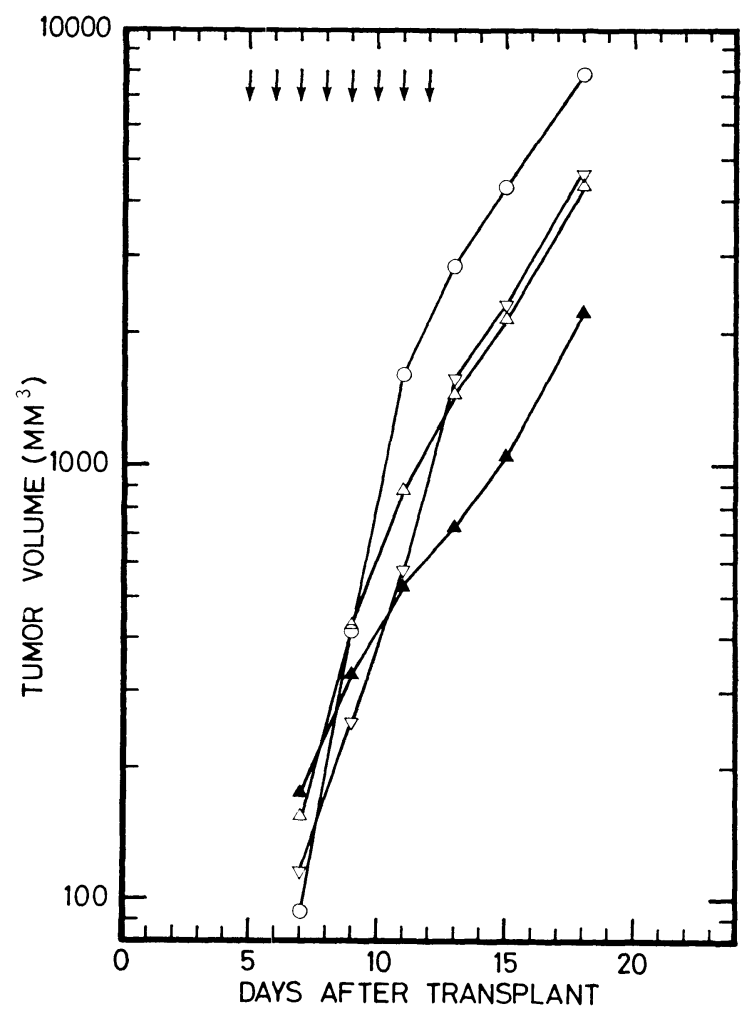

Fig. 2. Inhibitory effect of rHIL-2 and/or rIFN- $\beta$ on tumor growth (adenocarcinoma 755) in nude mice. The S.E.s of the mean were less than $31 \%$ of the mean values. Inhibition in combination group on 11 through 15 days after transplant was significantly greater than that in $r H I L-2$ group $(\mathrm{P}<0.05)$. $-\mathrm{O}-$, untreated control; $-\triangle-$, rHIL-2; $-\nabla-$, rIFN- $\beta ;-\Delta-$, rHIL-2+ rIFN- $\beta$.

Therefore, these results suggest that T-cell activation is important for the synergistic inhibitory effect of the combination of rHIL-2 and rIFN- $\beta$ in C57BL/6 mice. This synergistic inhibition in normal mice and additive inhibition in nude mice of tumor growth by the combination of rHIL-2 and rIFN- $\beta$ may offer a new possibility for the therapeutic application of these biological response modifiers.

Acknowledgements. This work was supported by Grants-in-Aid from the 
Ministry of Health and Welfare for Cancer Research and for Comprehensive 10-Year Strategy for Cancer Control, Japan.

\section{References}

1) Rosenberg, S. A. et al. (1985): Surgery, 98, 437-444.

2) Saijo, N. et al. (1984): J. Cancer Res. Clin. Oncol., 107, 157-163.

3) Kabelitz, D. et al. (1985) : Cell Immunol., 93, 38-45.

4) Djeu, J. Y. et al. (1979) : J. Immunol., 122, 175-181.

5) Brooks, C. G. et al. (1985) : ibid., 135, 1145-1152.

6) Herberman, R. B., and Holden, H. T. (1978): Adv. Cancer Res., 27, 305-377.

7) Haller, O. et al. (1977): Nature, 270, 609-611.

8) Habu, S. et al. (1981): J. Immunol., 127, 34-38.

9) Kuribayashi, K. et al. (1981): ibid., 126, 2321-2327. 